\title{
La escritura como herramienta de aprendizaje significativo: un cuasiexperimento en la clase de ciencias
}

\section{Writing as a tool for meaningful learning: A quasi-experiment in science class}

\author{
Alejandra ANDUEZA CORREA \\ Universidad Alberto Hurtado. Chile
}

Recibido: Octubre 2014

Evaluado: Noviembre 2014

Aceptado: Abril 2015

\section{Resumen}

Este artículo presenta nuevas evidencias para sostener la hipótesis de que la escritura es una herramienta efectiva para el aprendizaje significativo de conceptos científicos. Con este propósito, se llevó a cabo un cuasiexperimento con grupo experimental, grupo de control, pretest y postest, en una escuela pública de Santiago de Chile. Al grupo experimental se le aplicó una secuencia didáctica diseñada para aprender conocimientos disciplinares a partir de un proceso de composición textual que promueve explícitamente la dialéctica de la escritura; y al grupo de control se le aplicó una secuencia diseñada para aprender los mismos conocimientos disciplinares, pero a partir de estrategias didácticas tradicionales de las ciencias naturales. Mediante la aplicación del pretest y postest, se determinó que los estudiantes del grupo experimental aprendieron significativamente más conocimientos disciplinares que el grupo de control. Se concluye que la producción textual no solo debe ser desarrollada en la asignatura de lenguaje, sino en todas las áreas del currículum.

Palabras clave: escribir para aprender, dialéctica de la escritura, aprendizaje significativo, didáctica de las ciencias.

\begin{abstract}
This article presents new evidence to support the hypothesis that writing is an effective tool for meaningful learning of scientific concepts. With this purpose, a quasi-experiment was conducted with an experimental group, control group, pre-test and post-test in a public school in Santiago, Chile. The experimental group was given a teaching sequence designed to learn disciplinary knowledge from a process of textual composition that explicitly promotes the dialectic of writing; and the control group was given a sequence designed to learn the same disciplinary knowledge, but from traditional teaching strategies in the natural sciences. Through the enforcement of the pre-test and post-test, it was determined that students from the experimental group learned significantly more disciplinary knowledge than the control group. It is concluded that text production is an ability that should not only be developed in the subject of language, but in all other areas of the curriculum.
\end{abstract}

Keywords: writing to learn, dialectic of writing, meaningful learning, science teaching. 
El potencial de la escritura como herramienta de aprendizaje (Writing to learn) se ha venido discutiendo desde la década de los sesenta (Bereiter y Scardamalia, 1987; Britton, Burgess, Martin, McLeod, Rosen, 1975; Emig, 1977, entre otros); pero, a pesar de que está ampliamente aceptado el hecho de que la escritura es un medio eficaz para aprender, la evidencia que la investigación ha aportado en este sentido sigue siendo difusa y contradictoria. (Gradwohl Nash y Schumacher, 1991; Kieft, Rijlaarsdam, van den Bergh, 2008; Kieft, Rijlaarsdam y van den Bergh, 2006; Newell, 2006; Tynjälä, 1998). Esto se debe, por una parte, a que son escasos los estudios que miden de manera rigurosa (pre/postest) resultados entre grupos de comparación y, por otra, a que solo en algunas ocasiones se reportan diferencias estadísticamente significativas entre los grupos (Fry y Villagomez, 2012).

Por su parte, las investigaciones que sí han reportado resultados favorables, indican que la influencia de la escritura en el aprendizaje tiende a ser leve (Nevid, Pastva, McClelland, 2012; Stewart, Myers, y Culley, 2010). Sin embargo, a nuestro juicio, dichas investigaciones proponen una aproximación de escribir para aprender que no explota todo el potencial de la escritura como herramienta epistémica. En efecto, las tareas en las que se utiliza la composición textual como medio de aprendizaje suelen consistir en actividades relativamente simples como: redacciones breves sobre un tema de estudio, entradas en journals; protocolos de aprendizaje y reflexiones personales, entre otros (Fry y Villagomez, 2012; Nevid, Pastva, McClelland, 2012; Nückles, Hubner y Renkl, 2009; Tracie, Stewart, Myers, y Culley, 2010). En otras palabras, plantean actividades de escritura "desestructurada" en las que el estudiante escribe para comprender o clarificar conceptos científicos (McDermott, 2010), sin considerar las exigencias de una audiencia y de un género discursivo determinado.

En vista de lo anterior, la presente investigación busca ofrecer evidencia para sostener que la escritura favorece el aprendizaje cuando el proceso de composición textual promueve un proceso dialéctico entre los problemas de contenido y los problemas retóricos del texto, cuyo efecto es la transformación del conocimiento en la mente del escritor (Bereiter y Scardamalia, 1987 y 1992; Nückles, Hubner y Renkl, 2009). Esto no implica que la escritura facilite la memorización de grandes cantidades de información, por el contrario, partimos de la base de que la escritura favorece una comprensión conceptual significativa (Klein y Rose, 2010) y la capacidad de vincular nuevos conceptos con otros preexistentes, de acuerdo con la teoría ausubeliana del aprendizaje significativo.

Con este propósito se llevó a cabo un cuasiexperimento con grupo experimental, grupo de control, pretest y postest. El grupo experimental realizó una secuencia didáctica diseñada para aprender conocimientos disciplinares a partir de un proceso de composición textual. Dicho proceso estaba enmarcado en un proyecto de escritura que consistía en elaborar una revista de divulgación científica. El proceso de escritura fue diseñado con el propósito expreso de favorecer la dialéctica de la escritura con el objeto de promover la adquisición significativa de conocimientos. Por su parte, el grupo de control llevó a cabo una secuencia diseñada para aprender los mismos conocimientos disciplinares, pero a partir de estrategias didácticas tradicionales de las ciencias naturales. 


\section{Marco teórico}

\section{La dialéctica de la escritura}

El concepto dialéctica de la escritura es el término utilizado por Scardamalia y Bereiter para explicar cómo un escritor experto transforma su conocimiento a lo largo del proceso de producción textual (Scardamalia y Bereiter, 1985). Esta dialéctica es característica de un proceso de composición textual que los autores han descrito mediante el modelo transformar el conocimiento (knowledge transforming) utilizado por escritores expertos, en contraposición al modelo decir el conocimiento (knowledge telling) utilizado por escritores inexpertos.

El modelo transformar el conocimiento sostiene que el desarrollo del pensamiento y la transformación del conocimiento se producen en la mente del escritor a partir de la interacción entre el procesamiento del texto y del conocimiento. Esta interacción tiene lugar en un proceso de resolución de problemas integrado por dos espacios: el espacio de problemas retóricos y el espacio de problemas de contenido (Balgopal y Wallace, 2013; Klein y Rose 2010; Scardamalia y Bereiter, 1985).

El modo en que el escritor experto se enfrenta y resuelve una tarea de escritura fue descrito por Bereiter y Scardamalia (1985 y 1987) de la siguiente manera: luego de la representación mental, el escritor analiza el problema, estableciendo los propósitos y los objetivos que pretende conseguir mediante el texto. Una vez superada esta etapa, se enfrenta a dos aspectos de la composición escrita que debe resolver: qué va a escribir y cómo va a hacerlo. Lo primero se desarrolla en el espacio de problemas de contenido, donde se rescata la información pertinente almacenada en la memoria y se transfiere al espacio de problemas retóricos donde se resuelven los aspectos acerca de cómo verbalizarlo (de acuerdo con los propósitos y con la audiencia del texto, establecidos inicialmente). Este proceso puede suponer nuevos problemas respecto del contenido lo cual demanda al autor volver a reflexionar sobre el contenido proposicional del texto, buscar nueva información, analizar posibles ideas complementarias, entre otras muchas posibilidades.

A lo largo de este proceso, el escritor reflexiona acerca de lo que está escribiendo, considera las diferentes posibilidades de expresión de una idea, analiza cada una y su relación con las demás ideas del texto, verifica si lo escrito expresa el significado que quiere transmitir o si consigue su propósito, verifica si está de acuerdo o cree aquello que ha escrito, etc. Estas y otras operaciones cognitivas transforman la matriz ideacional del escritor de modo que, al finalizar el proceso de composición textual y gracias a la dialéctica propia de ese proceso, el escritor sabe más y/o comprende mejor aquello sobre lo que ha escrito.

\section{El aprendizaje significativo de conceptos}

El concepto de aprendizaje significativo (meaningful learning) se contrapone a la noción de aprendizaje memorístico (root learning). Mientras este último consiste en almacenar, de manera literal y arbitraria, grandes cantidades de información; aprender significativamente implica vincular de manera consciente conocimientos nuevos con 
conocimientos previos en la estructura cognitiva del sujeto que aprende (Ausubel, 2000; Novak; 2010). Este tipo de aprendizaje favorece la progresiva organización y complejización de las redes de significado, lo cual promueve una comprensión más profunda de los conceptos y de las relaciones que los conceptos establecen entre sí en dicha red. Lo anterior, además, permite que el acceso y la aplicación de los conceptos aprendidos sea más rápida y más eficiente (Novak, 2010; Romance y Vitale, 2011).

Los conocimientos adquiridos significativamente se almacenan en la memoria de una manera no literal y no arbitraria. Esto implica que el estudiante utiliza sus conocimientos previos como matriz ideacional y organizativa para incorporar el nuevo contenido proposicional, es decir, no retiene literalmente la información, sino que asimila su contenido proposicional estableciendo vínculos con otros contenidos proposicionales preexistentes y pertinentes. Desde esta perspectiva teórica, las estructuras cognitivas son redes de significado compuestas por un conjunto proposiciones interconectadas. Estas proposiciones son aseveraciones viables respecto de cómo son y cómo funcionan diversos aspectos del universo y están compuestas por dos conceptos relacionados entre sí (Novak, Mintzes y Wandersee, 2005a). En este sentido, la correcta adquisición de conceptos promoverá la creación de nuevas proposiciones significativas que vincularán conceptos entre sí. Este nuevo contenido, que ha establecido vínculos con los previos, se convierte en un contenido de la conciencia definido, diferenciado y claramente articulado, que enriquece la red de significados y amplía la matriz de aprendizaje (Ausubel, 2000). Además, el hecho de que un nuevo conocimiento haya sido aprendido significativamente supone que será menos vulnerable al olvido o la interferencia; en otras palabras, será más fácil de retener (Novak, 2010; Romance y Vitale, 2011).

\section{Objetivo e hipótesis}

El objetivo del presente estudio es demostrar experimentalmente, mediante la implementación de una secuencia didáctica diseñada para promover la dialéctica de la escritura, que la composición de textos es una herramienta eficaz para el aprendizaje significativo de conceptos científicos.

Se formuló la siguiente hipótesis: los estudiantes que escriben reflexivamente textos de divulgación científica adquieren significativamente más conocimientos disciplinares que los estudiantes que aprenden con un método tradicional de enseñanza de la asignatura Estudio y Comprensión de la Naturaleza.

\section{Estudio cuasiexperimental}

\section{Diseño}

Se diseñó un estudio cuasi-experimental (Campbell y Stanley, 1978), con un grupo experimental, un grupo de control, un pretest y un postest, sin equivalencia pre- 
experimental de muestreo. La variable dependiente fue el aprendizaje significativo de conceptos científicos y la variable independiente, el tipo de tratamiento recibido.

A ambos grupos se les aplicó un pretest para medir los conocimientos previos que tenían respecto de los conceptos que se iban a enseñar/aprender, un tratamiento de ocho sesiones de duración y un postest mediante el cual se midió el impacto de ese tratamiento en el aprendizaje de los estudiantes.

Con el objetivo de mantener constantes todas las variables, excepto el tipo de tratamiento recibido, se determinó que el grupo experimental y el de control debían ser dos cursos de un mismo nivel y de una misma escuela, con el objeto de controlar, dentro de lo posible, la equivalencia de los grupos en cuanto a edad, conocimientos previos, nivel socioeconómico y zona geográfica. Igualmente, se determinó que ambos cursos debían tener el mismo docente en la asignatura de Estudio y Comprensión de la Naturaleza para descartar esta variable como posible causante de las diferencias en el aprendizaje de ambos grupos.

\section{Participantes}

La investigación se llevó a cabo en una escuela pública de una zona céntrica de Santiago de Chile. Dicha escuela pertenece al grupo socioeconómico medio, en el que entre un $25 \%$ y un $50 \%$ de sus estudiantes se encuentra en estado de vulnerabilidad social.

Participaron dos cursos (mixtos) de octavo año básico (13-14 años). El grupo de control $(\mathrm{N}=43)$ y el grupo experimental $(\mathrm{N}=43)$. Ambos tenían el mismo profesor de la asignatura Estudio y Comprensión de la Naturaleza y él fue quien impartió las sesiones del grupo de control y del grupo experimental, con ayuda de la investigadora quien asistió en todo momento las sesiones.

\section{Procedimiento}

Antes de comenzar la intervención, se dio a conocer el material al profesor colaborador de modo que pudiera familiarizarse con él y realizar sugerencias de modificaciones. Luego, se llevó a cabo una reunión en la que se analizó detenidamente la versión final del material y se planificó el modo en que se llevaría a cabo la intervención. Este material consistía en dos secuencias didácticas (Álvarez, 2007): una para el grupo experimental y otra para el grupo de control. Para el tratamiento experimental, se elaboró una secuencia que orienta la realización de un proyecto de escritura (Camps, 2003; Camps y Vila i Santasusana, 2003) diseñado con la intención expresa de que los estudiantes aprendieran significativamente conceptos científicos mediante la producción de un artículo de divulgación científica (Andueza, 2011a y $2011 b$ ). Para esto, se diseñó una secuencia de actividades promueven la dialéctica de la escritura a lo largo de las diversas fases de producción textual (Scardamalia y Bereiter, 1985). En otras palabras, la secuencia propone un proceso de escritura a lo largo del cual los estudiantes componen individualmente un artículo de divulgación mediante un 
proceso que estimula una transferencia constante de problemas de contenido (relativos al contenido proposicional del texto) en problemas retóricos (relativos al género discursivo, el propósito y la audiencia, principalmente) y viceversa.

El diseño de dicho proceso se basó en dos modelos de producción textual: uno cognitivo -transformar el conocimiento- (Bereiter y Scardamalia, 1987 y 1992) y otro sociocognitivo, pragmalingüístico y didáctico (Didactext, 2003). En concreto, la secuencia didáctica propone una primera fase, de acceso al conocimiento, en la que se desarrollan actividades de investigación y de familiarización con el género discursivo (Bajtín, 1979; Bronckart, 2004). Las actividades de investigación implican aplicar estrategias de comprensión lectora con el propósito de leer para escribir (Solé, 2006). Por su parte, las actividades de familiarización con el género discursivo implican la lectura y el análisis del tipo de texto que los estudiantes mismos tendrán que escribir con el propósito de identificar sus características estereotípicas analizando, entre otras cosas, el modo en que se resuelven determinados problemas que la producción del propio texto plantea (Camps, 2003). Ambas actividades se realizan promoviendo una interacción dialéctica, de modo que el contenido proposicional está determinado por las características estereotípicas del género discursivo, por la audiencia y por el propósito del texto.

La segunda fase, de planificación, propone actividades para que los escritores predeterminen el curso de la acción hacia la consecución de un propósito. En esta etapa, se llevan a cabo actividades de dos tipos; primero, las que tienen que ver con la selección, organización y jerarquización de la información del texto y; segundo, las que tienen que ver con aspectos retóricos como el propósito y los destinatarios del mismo. Luego, se pide a los estudiantes que integren ambos aspectos en un plan de escritura que busca registrar la representación mental del texto que se han formado a lo largo de las actividades realizadas. Este plan de escritura representa las metas y submetas que se pretenden alcanzar mediante la escritura del texto, la audiencia a la que estará dirigido el texto, el género discursivo a cuyas normas se debe adecuar la composición, y el significado global que se quiere transmitir, entre otras posibilidades (Bereiter y Scardamalia, 1987; Hayes y Gradwohl, 1996).

Dicha representación mental del texto sirve de guía para orientar el proceso y la resolución de los problemas retóricos y de contenido que surgen a lo largo de la composición textual, ya que ayuda al estudiante a generar, seleccionar, jerarquizar y organizar el contenido proposicional que va a transmitir, de acuerdo a las características del género discursivo, la audiencia y el propósito del texto.

La tercera fase, textualización, orienta al estudiante respecto de cómo convertir el plan de escritura en un discurso plenamente elaborado. Durante la redacción del primer borrador, el escritor debe manejar una gran cantidad de variables de tipo semántico, sintáctico, gramatical, ortográfico y léxico, entre otros, con lo cual se sugiere a los alumnos que centren su atención producir el sentido del texto. Se hace hincapié en este último aspecto dado que tiene una gran incidencia en el aprendizaje, en vista de que las operaciones y formas de organización de la información requeridas por el género diseñado de acuerdo a los principios del aprendizaje significativo-, genera, en la 
estructura cognitiva del escritor, formas análogas de organización del contenido (Klein, 1999).

En concreto, el género discursivo propuesto para este proyecto de escritura organiza la información en torno a tres partes que cumplen diferentes funciones: primero, la introducción, que hace las veces de organizador avanzado (Ausubel, 2000); segundo, el desarrollo, que va aportando información cada vez más diferenciada, que se puede vincular a la que ya ha sido adquirida; y, tercero, una conclusión que resume y evalúa lo dicho. Por su parte el título y los subtítulos organizan el contenido en torno a focos temáticos; y los conceptos clave y cuadros sinópticos concilian las diversas maneras de denominar un fenómeno, con lo cual favorecen la construcción o la reelaboración de las estructuras conceptuales del estudiante.

El primer borrador es sometido a una revisión diferida (Van Steendam, Rijlaarsdam, Van den Bergh y Sercu, 2014), en la que los estudiantes deben discriminar qué debe ser evaluado en cada ítem -si aspectos retóricos o de contenido-, y deben ser capaces de valorar el nivel de logro de cada aspecto. Para esto se promueve que los estudiantes lean el texto que han escrito, comparen el texto real con el texto ideal (representación mental) para identificar disonancias, diagnosticarlas y operar sobre ellas (Bereiter y Scardamalia, 1987; Sommers, 1980) para subsanarlas, reescribiendo el texto.

El proyecto de escritura contempla, además, un proceso de edición -tomado en cuenta las correcciones de la última revisión- de diseño, diagramación y encuadernación de la revista. Esta edición contempla un procesador de textos que permita organizar el documento de acuerdo a los elementos paratextuales propios del género discursivo.

Para el tratamiento de control se diseñó una secuencia didáctica, pero, en este caso, basada en los métodos tradicionales de enseñanza-aprendizaje de ciencias, para lo cual se consultaron distintas propuestas de libros de texto y de otros materiales didácticos. En concreto, la secuencia didáctica para el grupo experimental proponía una serie de actividades para aprender conceptos relacionados con la hidrósfera, la litósfera y la atmósfera. Estas actividades consistieron en: lectura de textos informativos y resolución de cuestionarios, realización de un experimento para comprender el ciclo de condensación del agua y elaboración de un planisferio de las placas tectónicas, entre otras. El tratamiento de control se realizó teniendo en cuenta la importancia de: (1) minimizar el efecto Hawthorne haciendo que éste se reparta homogéneamente en ambos grupos; (2) evitar la difusión o imitación entre el grupo experimental y el de control; y (3) mantener constante en ambos grupos los conceptos científicos enseñados a lo largo de las sesiones.

En una primera sesión se aplicó el pretest a ambos grupos el mismo día y a la misma hora, con el propósito de asegurar que cualquier variable extraña afectara a los dos grupos por igual, de modo que las consecuencias que dichas variables pudieran tener en el desempeño de los estudiantes estuvieran repartidas en ambos grupos.

Una vez aplicado el pretest, se llevó a cabo el tratamiento experimental y el de control a lo largo de ocho sesiones (20 horas) cada grupo. Después de la intervención, 
se realizó el postest en las mismas condiciones que el pretest, se llevó a cabo una evaluación general de la intervención.

\section{Instrumento de medición}

Para medir cuánto aprendieron los estudiantes a partir del tratamiento recibido, se aplicó a los estudiantes un pre y un postest consistente en la realización de un mapa conceptual. Los mapas conceptuales son instrumentos de medición que reflejan el conocimiento conceptual de los estudiantes en un determinado momento, expresado bajo la forma de un conjunto de proposiciones interrelacionadas entre sí. Estas proposiciones son unidades básicas de significado y están compuestas por nodos -que representan conceptos-, líneas -que representan la relación entre esos conceptos- y etiquetas -que caracterizan el tipo de relación existente entre dichos conceptos- (Merril, 2012; Shavelson y Ruiz Primo, 2005).

En este sentido, son instrumentos idóneos para medir la adquisición significativa de conceptos, porque permiten analizar cuán bien organizadas están las estructuras de conocimiento en la mente del estudiante; y cuán válidas son las relaciones que se establecen entre esas estructuras (Edmonson, 2005), con lo cual, son capaces evidenciar el cambio progresivo de los estudiantes a lo largo del tiempo (Novak, Mintzes y Wandersee, 2005b; Trowbridge y Wandersee 1996).

Existen diversos estudios que prueban la validez de los mapas conceptuales como herramienta de evaluación del aprendizaje (McClure, Sonak y Suen, 1999; Wallace y Mintzes, 1990; entre otros), así como diversos métodos para elaborarlos. Se utilizó la técnica de elaboración poco dirigida (Shavelson y Ruiz Primo, 2005) por ser poco costosa y relativamente fácil de elaborar. El procedimiento consistió en proveer a los estudiantes de folios en blanco, de un conjunto de 35 conceptos, de tijeras, lápiz y pegamento. Los estudiantes tuvieron que organizar los conceptos e interrelacionarlos en el folio, luego pegarlos y establecer los vínculos y las etiquetas con lápiz. Los estudiantes tanto del grupo de control como del grupo experimental ya tenían experiencia en la elaboración de los mapas conceptuales, con lo cual el docente juzgó que no era necesario realizar una sesión de entrenamiento.

La investigadora evaluó la totalidad de los mapas conceptuales. Con el objetivo de asegurar que la corrección fuera ciega, se asignó aleatoriamente un código a cada mapa conceptual, los cuales se registraron antes de eliminar toda evidencia de autoría, de modo que, durante la corrección, no fuera posible distinguir si el mapa pertenecía al grupo experimental, al grupo de control o si era pre o postest.

Cada mapa conceptual fue evaluado con un método holístico y un método analítico denominado método relacional con mapa modelo (McClure et al., 1999). Para la evaluación holística se utilizó una escala del 1 al 7. Este método ofrece un buen indicio de la calidad general del mapa ya que consiste en evaluarlo globalmente de acuerdo al nivel de comprensión de los conceptos que evidencia (McClure et al., 1999).

Para la evaluación relacional con mapa modelo (método analítico) se utilizó un protocolo de puntuación (ver figura 1) que permite evaluar separadamente cada una de 
las proposiciones que pueden ser calificadas con un puntaje que va de 1 a 4 siguiendo el protocolo adaptado en la figura 1. (McClure et al., 1999). Los resultados del mapa se calcularon, entonces, sobre la base de la puntuación total (suma del puntaje de todas las proposiciones) y sobre la ganancia de proposiciones que asumieron los distintos valores.

La valoración de cada proposición dependió de cuán específica y acertada fuera la relación entre los dos conceptos que la forman. En este sentido, la gradación 1 -2 -3 -4 da cuenta de la calidad del aprendizaje del sujeto porque refleja el modo en que ha asimilado los conceptos.

\section{Tratamiento de los datos}

Los datos obtenidos a partir de la puntuación de los mapas conceptuales fueron analizados descriptiva e inferencialmente con el paquete estadístico SPSS. Primero, se calculó la media y la desviación típica de cada variable y, posteriormente, se aplicó la prueba de Mann-Whitney a las variables ordinales y la prueba $t$ a las variables continuas.

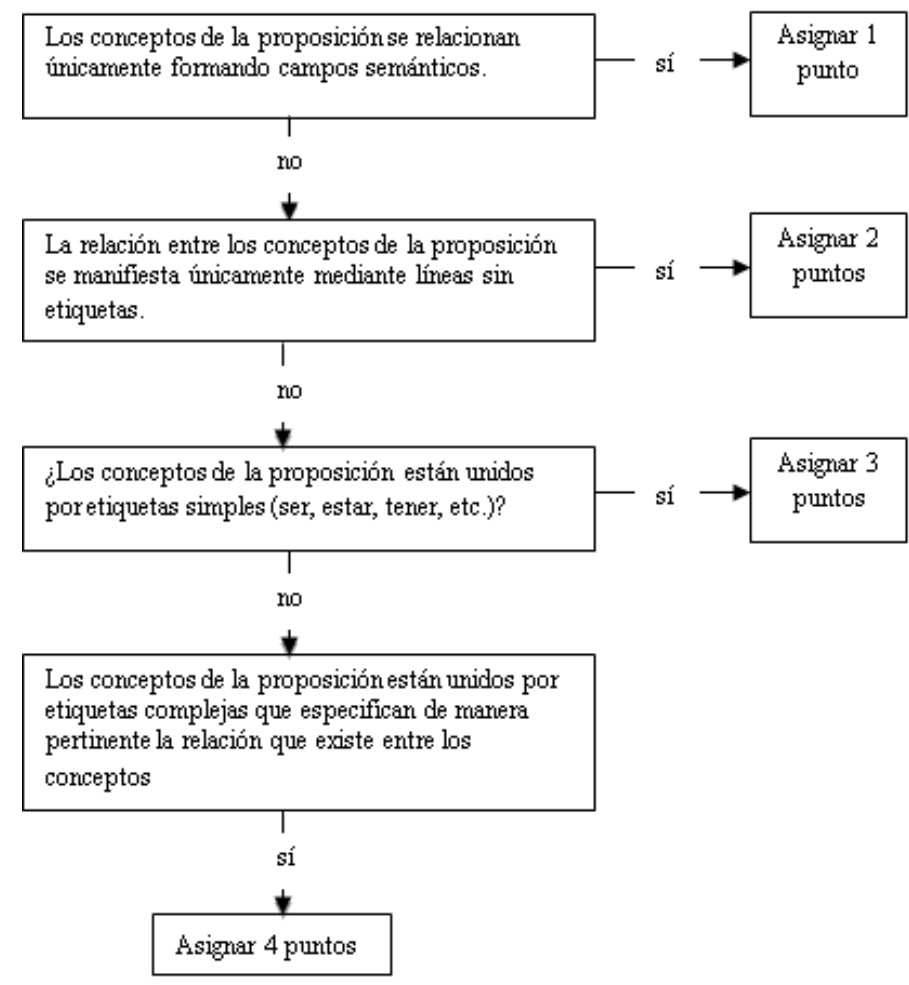

Figura 1. Protocolo para la puntuación de las proposiciones del mapa conceptual 


\section{Resultados}

\section{Evaluación del mapa conceptual con el método holístico}

En el pretest, el grupo experimental $(\mathrm{M}=3,97, \mathrm{DT}=1,06)$ obtuvo una puntuación menor en la variable ordinal "valoración holística del mapa conceptual" que el grupo de control $(\mathrm{M}=4,14, \mathrm{DT}=0,96)$. En cambio, en el postest, el grupo experimental $(\mathrm{M}=4,84, \mathrm{DT}=1,13)$ obtuvo una puntuación mayor que el grupo de control $(\mathrm{M}=4,14$, $\mathrm{DT}=0,79)$.

Posteriormente se aplicó la prueba de Mann-Whitney. Los resultados del pretest indican que los estudiantes del grupo experimental $(\mathrm{Mdn}=4)$ obtuvieron puntuaciones similares del grupo de control $(M d n=4) U=306,000 ; Z=-0,571, p=0,568$ y $r=0,07$. Por el contrario, los resultados del postest indican que los estudiantes del grupo experimental $(\mathrm{Mdn}=5)$ obtuvieron puntuaciones más altas que las del grupo de control $(\mathrm{Mdn}=4) \quad \mathrm{U}=221,000 ; \mathrm{Z}=2,221 ; \mathrm{p}=0,026$ y $\mathrm{r}=0,3$ y que esta diferencia sí es estadísticamente significativa.

\section{Evaluación del mapa conceptual con el método relacional: puntuación directa}

La puntuación directa corresponde al producto de la suma del valor de todas las proposiciones de un mapa conceptual. Se aprecia que, en el pretest, la media del grupo experimental $(M=41,09$. DT=18,61) es mayor que la media del grupo de control $(\mathrm{M}=37,38, \mathrm{DT}=16,02)$ al igual que en el postest (grupo experimental: $\mathrm{M}=54,88$, $\mathrm{DT}=21$ y grupo de control $(\mathrm{M}=44,62, \mathrm{DT}=17,35)$. ,

Se escogió la prueba t de student para verificar la diferencia de medias de ambos grupos. Para esto, en primer lugar, se comprobaron los supuestos de normalidad con la prueba Kolmogorov-Smirnov, (pretest $\mathrm{z}=1,023 ; \mathrm{p}=0.246$ y postest $\mathrm{z}=1.203$ y $\mathrm{p}=0.111$ ). En segundo lugar, se aplicó el test de Levene (pretest $\mathrm{F}=0,005 ; \mathrm{p}=0,944$ y para el postest: $\mathrm{F}=0,002 ; \mathrm{p}=, 966)$. Dichas prueban confirman normalidad y homocestadicidad.

La prueba $t$ indica que en el pretest no hay diferencias significativas entre la media del grupo experimental y la media del grupo de control $(t=0,749 ; p=0,457$ y $r=0,1)$. En el postest, tampoco se encontraron diferencias estadísticamente significativas entre las medias de ambos grupos $(\mathrm{t}=1.858 ; \mathrm{p}=0,069 \mathrm{r}=0,25)$.

\section{Evaluación del mapa conceptual con el método relacional: ganancia tipo de proposición}

En vista de que el valor asignado a cada proposición refleja el grado de comprensión de esa proposición, se calculó la ganancia de tipos de proposición para verificar si los alumnos habían elaborado más proposiciones de valor 3 y 4 en el postest respecto del pretest.

Respecto de la ganancia de proposiciones con valor 0, el grupo experimental obtuvo una media de ganancias de $1,16(\mathrm{DT}=4,53)$ y el grupo de control una media de $0,62(\mathrm{DT}=3,369)$. En cuanto a la ganancia de proposiciones con valor 1, el grupo 
experimental obtuvo una media de ganancias de $2,56(\mathrm{DT}=4,28)$ y el grupo de control una media de $-0,33$ ( $\mathrm{DT}=8,743)$. Por su parte, en la ganancia de proposiciones con valor 2, el grupo experimental obtuvo una media de 0,19 $(\mathrm{DT}=7,231)$ y el grupo de control una media de 3,0 (DT=9,508). En lo referido a la ganancia de proposiciones con valor 3 , el grupo experimental obtuvo una media de $-0,59(\mathrm{DT}=4,493)$ y el grupo de control una media de 0,9 (DT=2,879). Por último, en cuanto a la ganancia de proposiciones con valor 4, el grupo experimental obtuvo una media de 3,16 $(\mathrm{DT}=4,946)$ y el grupo de control una media de $-0,29(\mathrm{DT}=3,552)$

A continuación se aplicó la prueba t para verificar si la diferencia entre las medias de ambos grupos es estadísticamente significativa. Para esto, primero se comprobaron los supuestos de normalidad con la prueba Kolmogorov-Smirnov. Los resultados son los siguientes: ganancia de proposiciones con valor $0(z=0,72 ; p=0,641)$ ganancia de proposiciones con valor $1(\mathrm{z}=0,853 ; \mathrm{p}=0,461)$, ganancia de proposiciones con valor 2 $(\mathrm{z}=0,927 ; \mathrm{p}=0,356)$, ganancia de proposiciones con valor $3(\mathrm{z}=0,768 \mathrm{p}=0,596)$, ganancia de proposiciones con valor $4(\mathrm{z}=1,208 ; \mathrm{p}=0,108)$. De igual manera, se comprobó el supuesto de homocedasticidad con el test de Levene, con los siguientes resultados: ganancia de proposiciones con valor $0(\mathrm{~F}=0,028 ; \mathrm{p}=0,868)$, ganancia de proposiciones con valor $1(\mathrm{~F}=1,194 ; \mathrm{p}=0,280)$ ganancia de proposiciones con valor 2 $(\mathrm{F}=0,915 ; \mathrm{p}=0,343)$ ganancia de proposiciones con valor $3(\mathrm{~F}=1,400 ; \mathrm{p}=0,242)$ ganancia de proposiciones con valor $4(\mathrm{~F}=4,805 ; \mathrm{p}=0,033$, no se asume igualdad de varianza).

La prueba $t$ indica que no hay diferencias estadísticamente significativas en las medias de la ganancia de proposiciones de valor $0(\mathrm{t}=0,465, \mathrm{p}=0,644$ y $\mathrm{r}=0,06)$, valor 1 $(\mathrm{t}=1,608, \mathrm{p}=0,114$ y $\mathrm{r}=0,21)$, valor $2,(\mathrm{t}=-1,221, \mathrm{p}=0,228$ y $\mathrm{r}=0,16)$, ni valor 3 , $(\mathrm{t}=-$ $1,353, \mathrm{p}=0,228$ y $\mathrm{r}=0,19)$. Sin embargo, la diferencia de medias de la ganancia de proposiciones de valor 4 sí es estadísticamente significativa $(t=2,753, p=0,008$ y $\mathrm{r}=0,38)$. los resultados indican que el grupo experimental obtuvo una media de ganancia significativamente mayor de proposiciones con valor "4" -que evidencian una comprensión más profunda de los conceptos y de las relaciones que se establecen entre ellos.

\section{Discusión}

A partir de los resultados obtenidos, podemos decir que, como consecuencia del proceso de escritura, los estudiantes del grupo experimental obtuvieron un mayor aprendizaje significativo que los estudiantes del grupo de control. Consideramos que la diferencia en los aprendizajes del grupo de control y del grupo experimental se deben, al menos en parte, a que la secuencia didáctica colabora directamente con el proceso dialéctico que sucede cuando, durante la escritura, interactúan dos tipos de problema: los de contenido y los retóricos (Scardamalia y Bereiter, 1985).

En efecto, la secuencia didáctica del tratamiento experimental demanda al escritor que analice, reflexione y resuelva aspectos relativos a qué decir y al cómo decirlo a lo largo de cada una de las fases de la escritura, lo cual promueve una transferencia constante entre los problemas retóricos y de contenido que favorece la transformación 
del conocimiento. Consideramos que lo anterior es una diferencia clave entre la presente propuesta de escribir para aprender y las actividades que típicamente se proponen a los estudiantes en esta línea pedagógica, en la que se solicita redactar textos para reflexionar sobre algún contenido de aprendizaje, sin atender las exigencias de un género discursivo particular, ni de una audiencia concreta (Fry y Villagomez, 2012; Nevid, Pastva, McClelland, 2012; Nückles, Hubner y Renkl, 2009; Tracie, Stewart, Myers, y Culley, 2010). A nuestro juicio, dichas actividades, aunque valiosas, no explotan todo el potencial de la escritura como herramienta de aprendizaje ya que se centran en reflexionar sobre lo que ya se sabe, más que en generar significado en torno a ese conocimiento. Creemos que este proyecto de escritura, al promover la dialéctica de la escritura y proponer actividades que implican la adquisición de estrategias cognitivas y metacognitivas propias de la prodición textual, estimula la adquisición, generación y vinculación de ideas nuevas con ideas previas.

Por otro lado, consideramos que esta secuencia didáctica también favorece el aprendizaje significativo de conocimientos porque ofrece diversas instancias en las que el profesor puede detectar y subsanar malos entendidos y errores conceptuales que están generalizados entre sus estudiantes. De hecho, a lo largo de todas las actividades, los estudiantes tienen diversas oportunidades para tomar notas, realizar lluvias de ideas, redactar borradores, y, en general, expresar con sus propias palabras qué es lo que saben respecto del tema, es decir, evidenciar la estructura conceptual que tienen respecto de un área particular de conocimiento. Con esto, dan cuenta de los conceptos que han aprendido erróneamente o de los vínculos no apropiados que han establecido entre dichos conceptos. Por ejemplo, en los productos de las diversas fases del proceso, se pudo observar que los estudiantes tendían a asociar cualquier catástrofe natural terremotos, maremotos, etc.- con la contaminación y que estaba muy generalizada la idea de que dichos fenómenos suceden porque los seres humanos están destruyendo la Tierra. Lo anterior ofrece al docente, o, incluso, a un par más competente, la oportunidad de detectar malos entendidos, aclararlos y ayudar al estudiante a reelaborar los conceptos adquiridos erróneamente; y, además, en las sucesivas versiones del texto, es posible monitorear que esos malos entendidos hayan sido subsanados.

\section{Conclusiones}

Los resultados apoyan lo propuesto por Nückles, Hubner y Renkl (2009) que las actividades de escribir para aprender son más eficaces si se centran en desarrollar estrategias cognitivas y metacognitivas propias del proceso de producción textual, especialmente, si favorecen el proceso dialéctico. Consideramos que secuencias didácticas como la que se propone en este trabajo pueden ser muy útiles para, por una parte, aumentar la capacidad de los estudiantes de autorregular sus procesos de aprendizaje $\mathrm{y}$, por otra, para iniciarlos en las prácticas comunicativas y de investigación propias de los científicos. En este sentido, consideramos fundamental ofrecer, en la escuela, más oportunidades de escritura (Lerner, 2007) en las que los alumnos pueden constatar la estrecha relación que existe entre el uso y la producción de conocimiento y las estructuras retóricas mediantes las cuales ese conocimiento se transmite (Bazerman, Little, Bethel, Chavkin, Fouquette, y Garufis, 2005; Sanmartí, 
2007). En la práctica, las oportunidades reales de escritura en la escuela tienden a ser escasas, especialmente fuera de la clase de lengua. En vista de los resultados obtenidos, consideramos beneficioso integrar el desarrollo de secuencias didácticas diseñadas con el propósito de escribir para aprender en todas las asignaturas, no sólo porque beneficia el aprendizaje significativo de conocimientos disciplinares, sino que también porque promueve la familiarización -y progresivo dominio- de los géneros discursivos mediante los cuales cada disciplina genera y difunde el conocimiento.

\section{Limitaciones del estudio y prospectiva}

Si bien los resultados respaldan la hipótesis de que la escritura es una herramienta eficaz para promover el aprendizaje significativo, en vista de que se trata de un diseño cuasiexperimental, en el que los grupos nos son aleatorios, no es posible generalizar las conclusiones a toda la población. En este sentido, resulta fundamental aplicar estudios de este tipo a una mayor escala, que permitan verificar la eficacia de este modelo didáctico para escribir para aprender en diversos contextos.

\section{Referencias bibliográficas}

ÁLVAREZ, T (2007). Enseñar y aprender a escribir textos expositivos en colaboración en educación primaria. En T. Álvarez (Dir.), La competencia en comunicación lingüística en las áreas del currículo (pp 79 -102). Madrid: Ministerio de Educación y Ciencia.

ANDUEZA, A. (2011a). Escribir para aprender en la clase de ciencias: una propuesta didáctica y su experimentación en el aula. En M. Nuñez Delgado y J. Rienda Granada (Coords.), La investigación en Didáctica de la Lengua y la Literatura: situación actual y perspectivas de futuro (pp. 169-190) Madrid: Sociedad Española de didáctica de la lengua y la literatura.

ANDUEZA, A. (2011b) Escribir en diversas áreas del currículum: una propuesta didáctica para la clase de ciencias. Lenguaje y Textos, 33, 49-59.

AUSUBEL, D. (2000). Adquisición y retención del conocimiento: Una perspectiva cognitiva (G. Sánchez Trad.). Barcelona: Paidós.

BAJTÍN, M. (2008). Estética de la creación verbal (trad. de T. Bubnova). Buenos Aires: Siglo XXI. (Obra original publicada en 1979)

BALGOPAL, M Y WALLACE, A. (2013) Writing-to-Learn, Writing-toCommunicate, \&Scientific Literacy. The American Biology Teacher, 75, (3), 170175. DOI:10.1525/abt.2013.75.3.5

BAZERMAN, C., LITTLE, J., BETHEL, L., CHAVKIN, T., FOUQUETTE, D. Y GARUFIS, J. (2005). Reference guide to Writing across the curriculum. Indiana: Parlor Press LLC. 
BEREITER, C. Y SCARDAMALIA, M. (1987). The Psychology of Written Composition. New Jersey: Lawrence Erlbaum Associates.

BEREITER, C. Y SCARDAMALIA, M. (1992). Dos modelos explicativos del proceso de producción escrita. Infancia y Aprendizaje, 53, 43-64.

BRITTON, J., BURGESS, T., MARTIN, N., MCLEOD, A., ROSEN, H. (1975). The Development of Writing Abilities (11-18). Gran Bretaña: Macmillan Education.

BRONCKART, J. P. (2004). Actividad verbal, textos y discursos. Por un interaccionismo socio-discursivo. (V. Salvador y M. J. Carrión trads,).Madrid: Fundación Infancia y Aprendizaje.

CAMPBELL, D. Y STANTLEY, J. (1978). Diseños experimentales $y$ cuasiexperimentales en la investigación social. Buenos Aires: Amorrotu Editores.

CAMPS, A. (2003). Proyectos de lengua entre la teoría y la práctica. En A. Camps (Comp.) Secuencias didácticas para aprender a escribir (pp 33-46). Barcelona: Graó.

CAMPS, A. Y VILA I SANTASUSANA, M. (2003). Proyectos para aprender lengua. En A. Camps (Comp.). Secuencias didácticas para aprender a escribir (pp 47-50). Barcelona: Graó.

DIDACTEXT (2003). Modelo sociocognitivo, pragmalingüístico y didáctico para la producción de textos escritos. Didáctica (Lengua y Literatura), 15, 77-104.

EDMONSON, K. (2005). Assessing science understanding through concept maps. En J. Mintzes, J. Wandersee y J. Novak (Eds.). Assesing science understanding: A human contructivist view (pp 19-40). Estados Unidos: Elsevier Academic Press.

EMIG, J. (1977). Writing as a Mode of Learning. College Composition and Communication, 28 (2), 122-128.

FRY, S. W., Y VILLAGOMEZ, A. (2012). Writing to learn: benefits and limitations.College Teaching, 60(4), 170-175. DOI:10.1080/87567555.2012.697081

GRADWOHL NASH Y SCHUMACHER (1991). Conceptualizing and measuring knowledge change due to writing. Research in the Teaching of English, 25, 67 - 96.

HAYES, J.R. GRADWOHL, J. (1996). On the nature of planning in writing. En M. Levy y S. Ransdell .(Eds.) The Science of Writing (pp 29-55). New Jersey: Lawrence Erlbaum Associates.

KIEFT, M., RIJLAARSDAM, G. Y VAN DEN BERGH, H (2006). Writing as a learning tool: testing the role of students' writing strategies. European Journal of Psychology of Education, 21, 17-34.

KIEFT, M., RIJLAARSDAM, G. Y VAN DEN BERGH, H. (2008). An AptitudeTreatment interaction approach to writing-to-learn. Learning and Instruction, 18, 379-390. DOI:10.1016/j.learninstruc.2007.07.004 
KLEIN, P. D., Y ROSE, M. A. (2010). Teaching argument and explanation to prepare junior students for writing to learn. Reading Research Quarterly, 45(4), 433-461. DOI:d.x.doi.org/10.1598/RRQ.45.4.4

LERNER, N (2007). Laboratory Lessons for Writing and Science. Written Communication 24, 191-222. DOI:10.1177/0741088307302765

MCCLURE, J., SONAK, B. Y SUEN, H. (1999). Concept map assessment of classroom learning: reliability, validity and logical practicality. Journal of Research in Science Teaching, 36, 475-492.

MCDERMOTT, M. (2010) More Than Writing-to-Learn: Using Multimodal Writing Tasks in Science Classrooms. The Science Teacher. 77, 1, 32-36

MERRILL, M.L. (2012) The Nature of Third Grade Student Experiences with Concept Maps to Support Learning of Science Concepts. En Proceedings of the fifth conference on concept mapping. Valleta, Malta. Descargado de http://citeseerx.ist.psu.edu/viewdoc/download?doi=10.1.1.412.5457\&rep=rep1\&typ $\mathrm{e}=\mathrm{pdf}$

NEVID, J. S., PASTVA, A., Y MCCLELLAND, N. (2012). Writing-to-Learn Assignments in Introductory Psychology Is There a Learning Benefit?. Teaching of Psychology, 39(4), 272-275. DOI: 10.1177/0098628312456622

NEWELL, G. (2006). Writing to learn: how alternative theories of school writing account for student performance. En C. MacArthur, S. Graham y J. Fitzgerald (Eds.). Handbook of writing research (pp 235-247). New York: The Guildford Press.

NOVAK, J. (2011) "Learning, creating, and using knowledge: Concept maps as a facilitative tools in schools and corporations". Journal of e-learning and Knowledge Society, 6 (3) 21-30.

NOVAK, J., MINTZES, J. Y WANDERSEE, J. (2005a). Learning, teaching and assessment: A human constructivist perspective. En J. Mintzes, J. Wandersee, y J. Novak (Eds.) Assessing Science Understanding. A human contructivist view (pp 113). San Diego: Elsevier Academic Press,

NOVAK, J., MINTZES, J., Y WANDERSEE, J. (2005b). Epilogue: on ways of assessing science understanding. En J. Mintzes, J. Wandersee, y J Novak. (Eds.) Assessing Science Understanding. A human contructivist view (pp 355- 374). San Diego: Elsevier Academic Press.

NÜCKLES, M., HÜBNER, S., Y RENKL, A. (2009). Enhancing self-regulated learning by writing learning protocols. Learning and instruction, 19(3), 259-271. DOI:10.1016/j.learninstruc.2008.05.002.

ROMANCE, N. R., Y VITALE, M. R. (2013). A Research-Based Instructional Model for Integrating Meaningful Learning in Elementary Science and Reading Comprehension. En N. Stein y S. Raudenbush (Eds.) Developmental Cognitive Science Goes to School (pp 127-42). New York: Routledge. 
SANMARTÍ, N. (2007). Hablar, leer y escribir para aprender ciencia. En T. Álvarez (dir.) La competencia en comunicación lingüística en las áreas del currículo (pp 103 -128). Madrid: Ministerio de Educación y Ciencia.

SCARDAMALIA, M. Y BEREITER, C. (1985). Development of dialectical processes in composition. En B. Stierer y J. Maybin (Eds.). Literacy, language and learning in educational practice (pp 295-309). Gran Bretaña: Multilingual Matters y The Open University.

SHAVELSON, R. Y RUIZ-PRIMO, A. (2005). On the psychometrics of assessing science understanding. En J. Mintzes, J. Wandersee, y J Novak. (eds.) Assessing science understanding: A human contructivist view (pp 303-341). San Diego: Elsevier Academic Press.

SOLÉ, I. (2006). Estrategias de lectura. Barcelona: Graó.

SOMMERS, N. (1980). Revision Strategies of Student Writers and Experienced Adult Writers. College Composition and Communication, 31 (4), 378-388.

STEWART, T. L., MYERS, A. C., Y CULLEY, M. R. (2009). Enhanced learning and retention through "writing to learn" in the psychology classroom. Teaching of Psychology, 37(1), 46-49. DOI: 10.1080/00986280903425813

TROWBRIDGE, J. Y WANDERSEE, J. (1996). How do graphics presented during college biology lessons affect students' learning?: A concept map analysis. Journal of College Science Teaching, 26, 54-57.

TYNJÄLÄ, P. (1998). Writing as a Tool for Constructive Learning: Students’ Learning Experience During an Experiment. Higher Education, 36, 209-230.

VAN STEENDAM, E., RIJLAARSDAM, G. C. W., VAN DEN BERGH, H. H., Y SERCU, L. (2014). The mediating effect of instruction on pair composition in L2 revision and writing. Instructional Science, 42(6), 905-927.

WALLACE, J. Y MINTZES, J. (1990). The concept map as a research tool: exploring conceptual change in biology. Journal of Research in Science Teaching, 27, 1033 1052.

\section{Correspondencia con la autora}

Alejandra ANDUEZA CORREA

Alameda 1869, oficina 412,

Santiago - Chile

e-mail: aandueza@uahurtado.cl 